\title{
Pulmonary Lymphoepithelioma-Like Carcinoma
}

\section{Treated with Immunotherapy or Chemotherapy: A Single Institute Experience}

This article was published in the following Dove Press journal: OncoTargets and Therapy

\section{Yang $\mathrm{Fu}^{*}$ \\ Yue Zheng* \\ Pei-Pei Wang (D) \\ Yue-Yun Chen \\ Zhen-Yu Ding}

Department of Biotherapy, Cancer Center, West China Hospital, West China Medical School, Sichuan University, Chengdu, People's Republic of China

*These authors contributed equally to this work
Correspondence: Zhen-Yu Ding Department of Biotherapy, Cancer Center, West China Hospital, Sichuan University, Chengdu, 61004I, People's Republic of China

$\mathrm{Tel}+8602885422562$

Fax +860288516 4059

Email dingzhenyu@scu.edu.cn
Background: Lymphoepithelioma-like carcinoma (LELC) is a rare malignant tumor of the lung. It is related to EB virus infection. Epidermal growth factor receptor (EGFR) and anaplastic lymphoma kinase (ALK) are rarely found in this disease, while high level programmed cell death ligand 1 (PD-L1) expression is observed. Here a series of patients with advanced LELC treated with immunotherapy were summarized.

Methods: This retrospective, observational study was conducted in patients who were pathologically confirmed, metastatic or recurrent LELC patients. Patients were prescribed with either chemotherapy or immunotherapy, according to treating physicians' discretion.

Results: A total of 27 patients were included in our study, 10 with immunotherapy (ICI group) and 17 with chemotherapy (Chemo group). The objective response rates (ORR) of the two groups were $80.0 \%$ and $70.5 \%(\mathrm{p}=0.678)$, and disease control rates (DCR) were $100 \%$ and $88.2 \%(p=0.516)$. However, the response depth was better in the ICI group. Although the cohort of patients in the ICI group was in a disadvantageous state (both up-front and salvage), the progression-free survival (PFS) was much longer (15.0 and $7.9 \mathrm{~m}, \mathrm{p}=0.005$ ). The 1-year PFS rate in the ICI group was also much higher $(40 \%$ and $5.9 \%, \mathrm{p}=0.047)$. Conclusion: This study implicated the high efficiency of ICI therapy in this disease. Keywords: lymphoepithelioma-like carcinoma, ICIs, PD-L1, immunotherapy

\section{Introduction}

Pulmonary lymphoepithelioma-like carcinoma (LELC) is a rare malignant tumor of the lung. It belongs to the category of non-small cell lung cancer (NSCLC) with an incidence of $\leq 0.7 \%$ of all NSCLC. ${ }^{1-4}$ According to the World Health Organization Classification of Tumors of the Lung (2015), LELC was classified into "other and unclassified carcinomas". Morphological features are similar to those of undifferentiated nasopharyngeal carcinoma. ${ }^{5,6}$ Typically, poorly differentiated tumor cells and a large number of lymphocyte infiltration can be observed. ${ }^{7,8}$ LELC may be related to EB virus infection, and has a higher incidence in Southeast Asia, such as Guangdong, Hongkong, Taiwan, and Singapore, than in Europe and the US. ${ }^{9,10}$ Epidermal growth factor receptor (EGFR) and anaplastic lymphoma kinase (ALK), the most sensitive driver genes to targeted therapy in NSCLC, are rarely found in this disease. ${ }^{11-13}$ Recent studies showed a higher proportion of programmed cell death ligand 1 (PD-L1) positivity tumor cells in LELC than adenocarcinoma and squamous cell carcinoma. ${ }^{8,11,13}$ No standard-of-care for this disease is established, but the current therapy often contains multimodality treatments, such as radio- or 
chemo-therapy. ${ }^{14-16}$ Hereto, immunotherapy has been suggested in anecdotal case reports, ${ }^{17-22}$ and apparently more cases are needed to confirm its efficacy. Here we summarized our experience of patients with advanced LELC treated with immunotherapy.

\section{Patients and Methods}

\section{Patients}

This retrospective, observational study was conducted in our hospital. This study was approved by the Ethics Committee on Biomedical Research (No. 669). All admitted lung cancer patients were screened through the Hospital Information System from January 2012 to June 2020. Enrolled patients were pathologically confirmed, metastatic or recurrent LELC patients. PD-L1 expression was centrally stained and assessed with antibody $22 \mathrm{C} 3$.

\section{Treatment}

Patients were prescribed chemotherapy or immunotherapy. Immunotherapy drugs included pembrolizumab (Merck \& Co., Inc., Whitehouse Station, NJ, USA), or sintilimab (Innovent, China), or nivolumab (BristolMyers Squibb, New York, NY, USA). The chemotherapy regimen consisted of either platinum-based doublet chemotherapy, or albumin paclitaxel or pemetrexed monotherapy. The tumor responses were assessed the RECIST 1.1. and adverse events were graded on CTC AE 4.0. Tumors were assessed every 2 months radiographically, including computed tomography of the chest and upper abdomen, magnetic resonance imaging of the head, and bone scintigraphy. Tumor response was evaluated as complete response (CR), partial response (PR), stable disease (SD), or progression disease (PD), according to RECIST 1.1. The PFS was defined as the duration from the initiation of the therapy to the date of disease progression, intolerable side effects, or death from any cause.

\section{Statistics}

Statistical analysis was performed using SPSS version 22.0 (IBM Corp., Armonk, NY, USA). The quantitatives were compared using chi-square test and Fischer's exact test according to Cochran's rule. The Kaplan-Meier curves were used to compare survival. All P-values were based on a two-tailed hypothesis, and statistical significance was assumed if $\mathrm{p}<0.05$.

\section{Results \\ Clinical Characteristics}

A total of 27 patients were included. Ten and 17 patients received immunotherapy (either alone or in combination with other therapies, ICI group) or chemotherapy (Chemo group), respectively (Figure 1, Table 1). Of notice, immunotherapy was administered either as up-front or salvage therapy (1st-line $\mathrm{n}=4, \geq 2$ nd-line $\mathrm{n}=6$ ), while chemotherapy was used in the 1 stline therapy. The median age was 57.2 and 55.2 years in each group. Some patients received surgery before (3/10 in ICI group, 3/16 in Chemo group). Extrapulmonary metastases were common ( $80 \%$ in ICI group, $53 \%$ in Chemo group). In the ICI group, PD-L1 expression was assessed in 9 patients. In the whole cohort, no EGFR or ALK aberration was detected in any patients, and FGFR3 gene amplification was found in only 1 case (ICIs group).

\section{Treatment}

In the ICI group, 3 drugs were prescribed, including pembrolizumab $(n=3)$, nivolumab $(n=3)$, and sintilimab $(n=4)$. These ICIs were either combined with chemotherapy $(n=3)$ or small molecular antiangiogenic agents $(n=3)$, or as a single agent $(n=4)$. The median cycle of administration was 7.5 (range: $2-25$ ).

\section{Efficacy}

The objective response rates (ORR) of the two groups were $80.0 \%(8 / 10)$ and $70.5 \%(12 / 17)$, respectively, with no significant difference $(\mathrm{p}=0.678)$. The disease control rates (DCR) were also similar $(100 \%$ and $88.2 \%$, $\mathrm{p}=0.516$ ). The response depth was better in the ICI group.

The median follow-up time was 22.4 months (range 3.5-60.9 months). A longer PFS was achieved in the ICI group than the Chemo group (15.0 and $7.9 \mathrm{~m}, \mathrm{p}=0.005$, Figure 2). The 1-year PFS rate in the ICI group was also much higher $(40 \%$ and $5.9 \%, \mathrm{p}=0.047)$. During treatment course, 4 patients had disease progression in ICI group, compared to 13 in Chemo group. Till the time of preparation of this manuscript (Aug 2020), persistent PR ( $n=5$ and 3) and SD ( $\mathrm{n}=1$ and 1) were maintained in ICI and Chemo groups. Distant metastases including intra- and extra-pulmonary in ICI (2/10 and $1 / 10)$ were comparable to those in Chemo group (4/17 and $5 / 17, \mathrm{p}=0.42)$.

\section{Toxicities of Immunotherapy}

Two patients developed grade 1 hypothyroidism. After thyroxine replacement, both gradually recovered without 


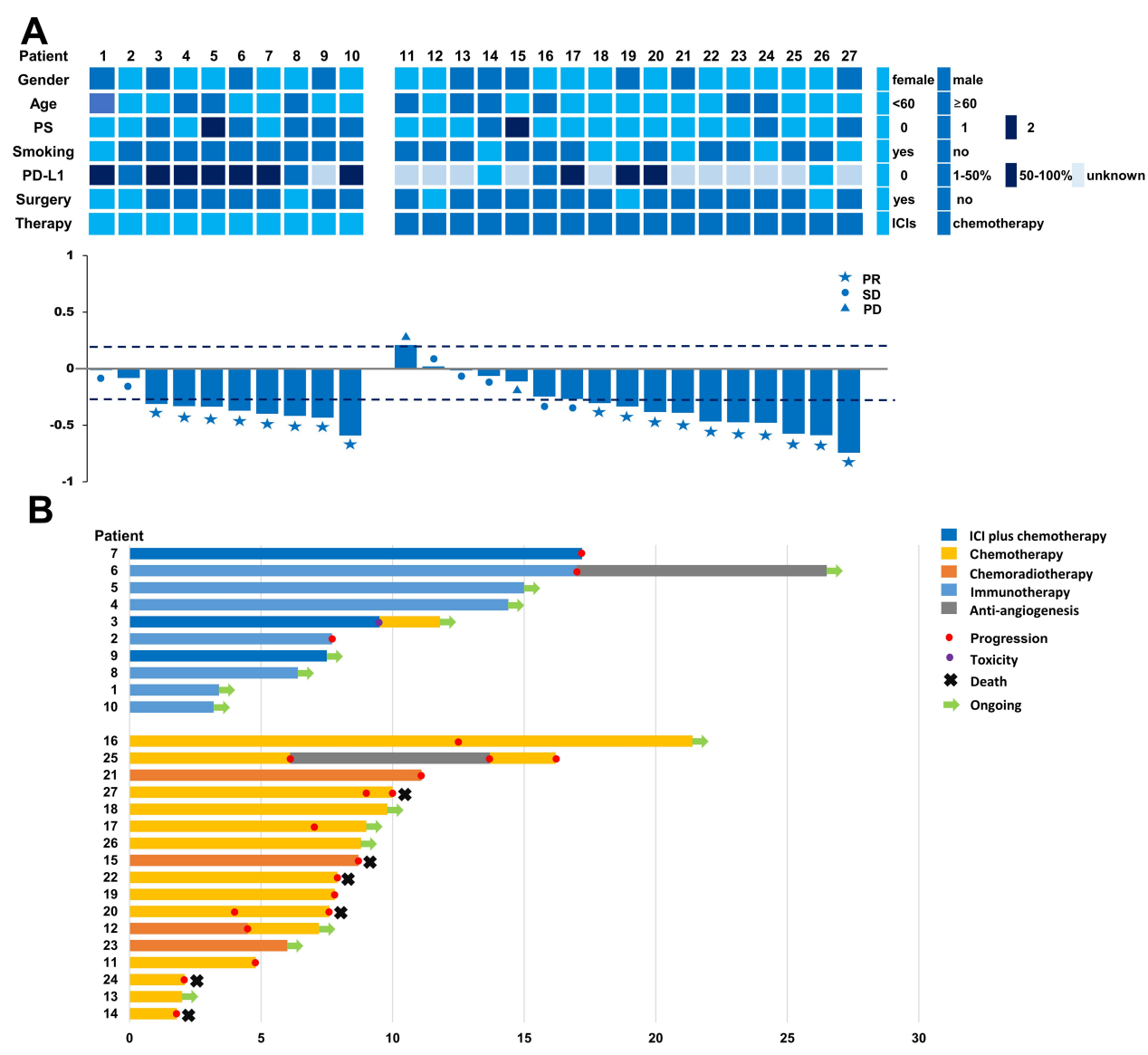

Figure I Tumor response of each patient receiving ICl or chemotherapy (A). Time course of treatment in each patient (B).

delay in therapy. One patient achieved a PFS of 3.7 mon, and another was maintained in PR for 6.4 mon. One patient had severe (grade 4) immune-related multiorgan failure (below).

\section{Patients Report}

Patient 4 was a 63-year-old female. She was admitted because of gradual onset of productive cough, and intermittent hemoptysis, accompanied by a slight fever $(\leq 38.5 \quad$ oC) without any obvious causes. A comprehensive workshop consisting of enhanced chest CT, PET-CT, and MRI of the brain, showed a mass in the left lung lower lobe with hilar mediastinal lymph node enlargement, left main bronchus and lower lobe bronchus invasion, and the lesions of right humerus and pleural. Bronchoscopy and pathology revealed poorly differentiated NSCLC in the lower left main bronchus, Immunohistochemistry (IHC) staining demonstrated PCK (+), p63 (+), CK5/6 (+), TTF-1 (-), EBER1/ 2-ISH (+), PDL1 (+,70\%), ALK-V (-) and ros-1 (-). She was diagnosed as LELC in the left lung with metastases to the mediastinal and hilar lymph nodes, pleura and right humerus (cT4N2M1c, stage IVb). No EGFR or ALK aberrations were detected in the next generation sequencing of her tumor.

She was prescribed with 2 cycles of pemetrexed plus pembrolizumab $(2 \mathrm{mg} / \mathrm{kg})$ combination therapy. But an enlargement of the tumor in the left lung was shown in the following CT scan. Radiotherapy at the dose of $6 \mathrm{gy} / 3 \mathrm{f}$ was given concurrently with pembrolizumab as salvage therapy, and pembrolizumab was used regularly as maintenance therapy. After the 3 cycles of pembrolizumab, the size of the tumor and the lesions of the lymph node became smaller (Figure 3). After 1 year of therapy, the patient sustained remission without further complaints (sustained PR).

Patient 3 was a 55-year-old never smoking male. He came to our hospital and complained of unrelenting cough for 2 months with occasional hemoptysis. Enhanced CT of the chest showed an irregular mass in the middle lobe of the right lung, and multiple enlarged lymph nodes in the mediastinum and right hilum. He 
Table I The Demographic Feature of Enrolled Patients

\begin{tabular}{|c|c|c|c|c|c|c|c|c|c|c|}
\hline Patient & Gender & Age & PS & Smoking & PD-LI & Surgery & TNM & Stage & Line & Therapy \\
\hline 1 & M & 68 & 0 & yes & $80 \%$ & yes & rTONOMIC & IVB & 2nd & Sintilimab \\
\hline 2 & $\mathrm{~F}$ & 56 & 0 & no & $30 \%$ & yes & rTIbNOMIb & IVB & 2nd & Pembrolizumab \\
\hline 3 & M & 55 & I & no & $90 \%$ & no & T4N2Mlc & IVB & Ist & $\begin{array}{c}\text { Pembrolizumab+ Nab-paclitaxel+ } \\
\text { carboplatin }\end{array}$ \\
\hline 4 & $\mathrm{~F}$ & 63 & 0 & no & $70 \%$ & no & T4N2MIb & IVB & Ist & Pembrolizumab \\
\hline 5 & $\mathrm{~F}$ & 70 & 2 & no & $90 \%$ & no & T4N3Mla & IVA & 4th & Nivolumab+ Anlotinib \\
\hline 6 & M & 46 & I & no & $60 \%$ & no & T3N3MIC & IVB & 5 th & Nivolumab+ Apatinib \\
\hline 7 & $\mathrm{~F}$ & 56 & 0 & no & $80 \%$ & no & T4N2MIb & IVB & 2nd & Nivolumab+ Docetaxel \\
\hline 8 & $\mathrm{~F}$ & 61 & I & no & $40 \%$ & yes & rT3N2MIc & IVB & 2nd & Sintilimab+ Anlotinib \\
\hline 9 & $\mathrm{~F}$ & 54 & I & no & unknown & no & TIN3MIC & IVB & Ist & $\begin{array}{c}\text { Sintilimab+ Nab-paclitaxel+ } \\
\text { Carboplatin }\end{array}$ \\
\hline 10 & M & 43 & 0 & no & $80 \%$ & no & T3N3M0 & IIIC & Ist & Sintilimab \\
\hline 11 & $\mathrm{~F}$ & 76 & 0 & no & unknown & no & T4N2MIc & IVB & Ist & Nab-paclitaxel \\
\hline 12 & $\mathrm{~F}$ & 58 & 0 & no & unknown & yes & rTON3MIa & IVA & Ist & Nedaplatin+ Fluorouracil+ RT \\
\hline 13 & M & 77 & 0 & no & unknown & no & T3N2M0 & IIIB & Ist & Pemetrexed \\
\hline 14 & $M$ & 63 & $\mathrm{I}$ & yes & 0 & no & T4NIMIa & IVA & Ist & Pemetrexed+Cisplatin \\
\hline 15 & M & 52 & 2 & no & unknown & no & T4N3Mlb & IVB & Ist & Gemcitabine+ Cisplatin+ RT \\
\hline 16 & $\mathrm{~F}$ & 60 & 0 & no & $20 \%$ & no & $\mathrm{T} 2 \mathrm{bN} 2 \mathrm{MIb}$ & IVB & Ist & Paclitaxel+ Cisplatin \\
\hline 17 & $\mathrm{~F}$ & 52 & 0 & no & $50 \%$ & no & T2N3MIb & IVB & Ist & Gemcitabine+ Cisplatin \\
\hline 18 & $\mathrm{~F}$ & 50 & 0 & yes & unknown & no & T4N3Mla & IVA & Ist & Nab-paclitaxel+ Cisplatin \\
\hline 19 & M & 32 & 0 & yes & $65 \%$ & yes & rT2NOMIa & IVA & Ist & Gemcitabine+ Cisplatin \\
\hline 20 & $\mathrm{~F}$ & 55 & 0 & no & $85 \%$ & no & $\mathrm{T} 4 \mathrm{~N} 2 \mathrm{Mla}$ & IVA & Ist & paclitaxel+ Carboplatin \\
\hline 21 & M & 47 & 0 & yes & unknown & no & T4N3M0 & IIIC & Ist & $\begin{array}{c}\text { Fluorouracil+ Cisplatin+ } \\
\text { Bevacizumab+ RT }\end{array}$ \\
\hline 22 & $\mathrm{~F}$ & 49 & 0 & no & unknown & no & T4NIMIC & IVB & Ist & paclitaxel liposome +Cisplatin \\
\hline 23 & $\mathrm{~F}$ & 63 & 0 & no & unknown & no & T4N2MIc & IVB & Ist & Paclitaxel+ Cisplatin+ Endostar+ RT \\
\hline 24 & $\mathrm{~F}$ & 62 & $\mathrm{I}$ & yes & unknown & no & T4N3MIb & IVB & Ist & Paclitaxel+ Cisplatin \\
\hline 25 & $\mathrm{~F}$ & 52 & 0 & no & unknown & no & T4N3MIb & IVB & Ist & Docetaxel+ Nedaplatin \\
\hline 26 & $\mathrm{~F}$ & 48 & 0 & no & 0 & yes & rT4N2Mla & IVA & Ist & $\begin{array}{c}\text { Pemetrexed }+ \text { Cisplatin+ Endostar }+ \\
\text { Anlotinib }\end{array}$ \\
\hline 27 & M & 44 & I & yes & unknown & no & T4N3MIb & IVB & Ist & Gemcitabine+ Cisplatin \\
\hline
\end{tabular}

Abbreviations: F, female; $M$, male.

received Endobronchial Ultra-sound guided hilar and bilateral mediastinal lymph nodes, liver metasTransbronchial Needle Aspiration (EBUS-TBNA) of tasis (cT4N3M1c, stage IVb).

4R lymph node. According to pathology and imaging,

His tumor had high PD-L1 expression (90\%). Two he was diagnosed LELC of the right lung, with right cycles of pembrolizumab (200mg) combined with 


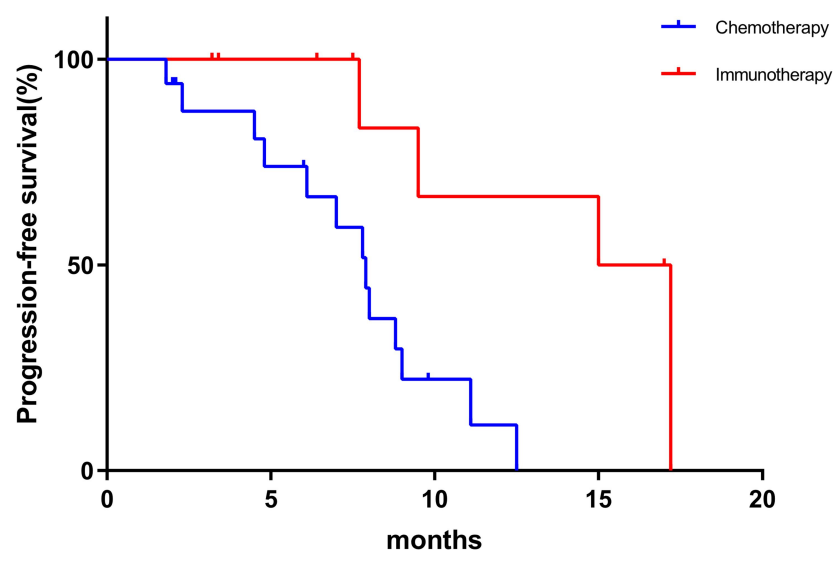

Figure 2 PFS in Chemo group was inferior to that in $\mathrm{ICl}$ group.

chemotherapy (albumin-bound paclitaxel 460mg, carboplatin $650 \mathrm{mg}$ every 3 weeks) were prescribed and his tumor achieved PR.

However, before the initiation of cycle 3 , he had a significant up-rise of transaminase, myocardial enzymogram, and other biochemical components (Figure 4). Left ventricular systolic function was normal (EF 72\%) in echocardiography, and no obvious abnormality was shown on cardiac MRI. He was diagnosed as immune-related multi-organ damage, including myocarditis, myositis, and acute liver injury. After intense methylprednisolone $(2 \mathrm{mg} / \mathrm{kg})$ therapy, all these biochemical parameters gradually resolved. Unfortunately, he developed severe bacterial and fungal pneumonia. During this period, no anti-tumor treatment was given, which contributed to his slightly enlarged tumor.

\section{Discussion}

There was no consensus on the treatment of advanced LELC. Although fluorouracil was suggested before the year of $2000,{ }^{14}$ the regimen gradually switched to paclitaxel/gemcitabine plus cisplatin. ${ }^{15,23}$ Targeted therapy revolutionizes the standard-of-care for those with NSCLC harboring sensitive gene mutations, while both EGFR and ALK mutations were hardly found in LELC. ${ }^{11-13}$ Interestingly, several lines of evidence support the role of immunotherapy for treatment of this disease.

\section{EBV}

Almost all patients of LELC had positive staining for EBER in fluorescence in situ hybridization. High
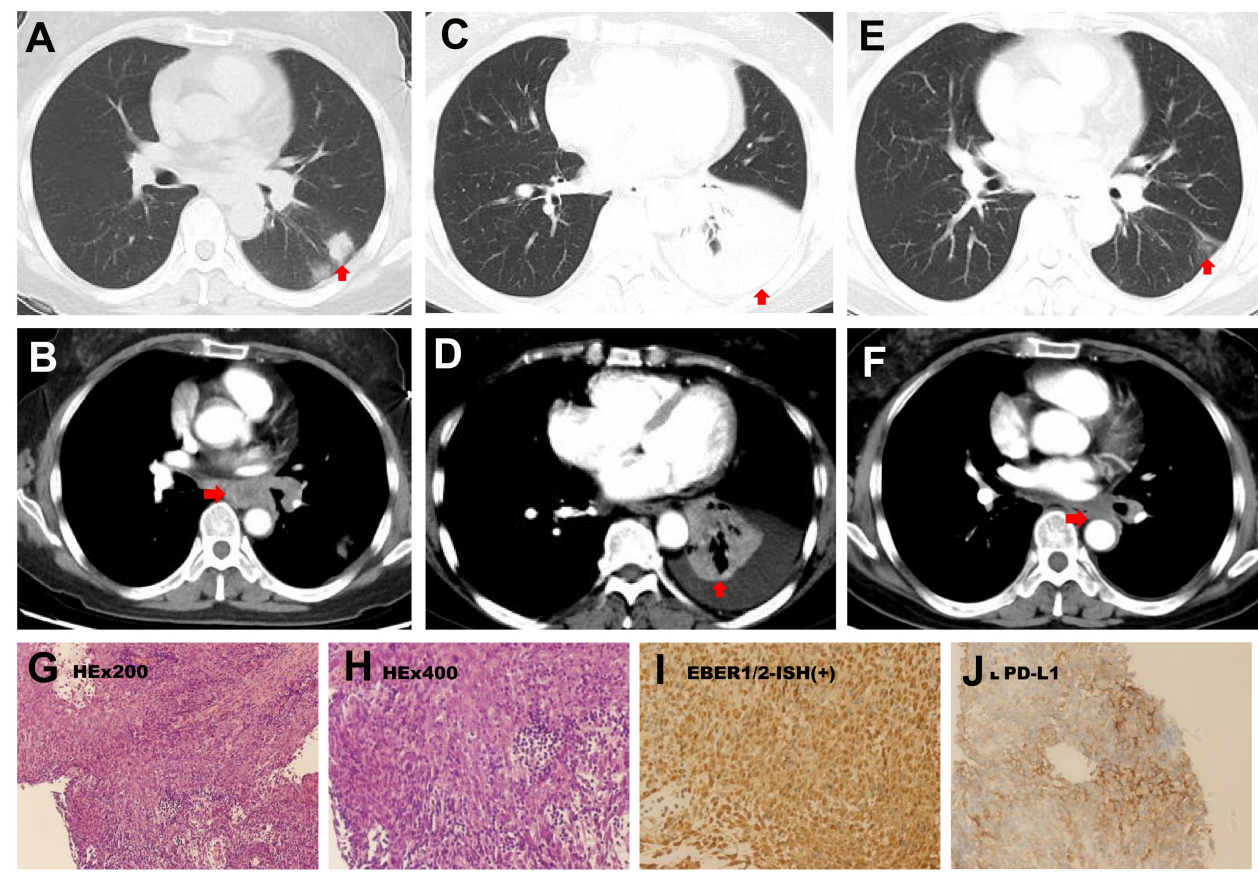

Figure 3 The patient had a mass in his left lung lower lobe and enlarged mediastinal lymph nodes at base line (A and B). After 2 cycles of chemotherapy and immunotherapy, her mass increased in size, accompanying with appearance of pleural effusion ( $\mathbf{C}$ and $\mathbf{D})$. When radiotherapy and immunotherapy were given, shrunken tumor and lymph nodes were observed (E and $\mathbf{F})$. Carcinoma cells in the background of infiltrating lymphocytes ( $\mathbf{G}$ and $\mathbf{H})$. In Situ Hybridization showed positive Epstein-Barr Virus-encoded RNA (I). Programmed Cell Death Ligand I staining by immunohistochemistry (J). Red arrow in Figure 3 (A-F) represent the tumor. 


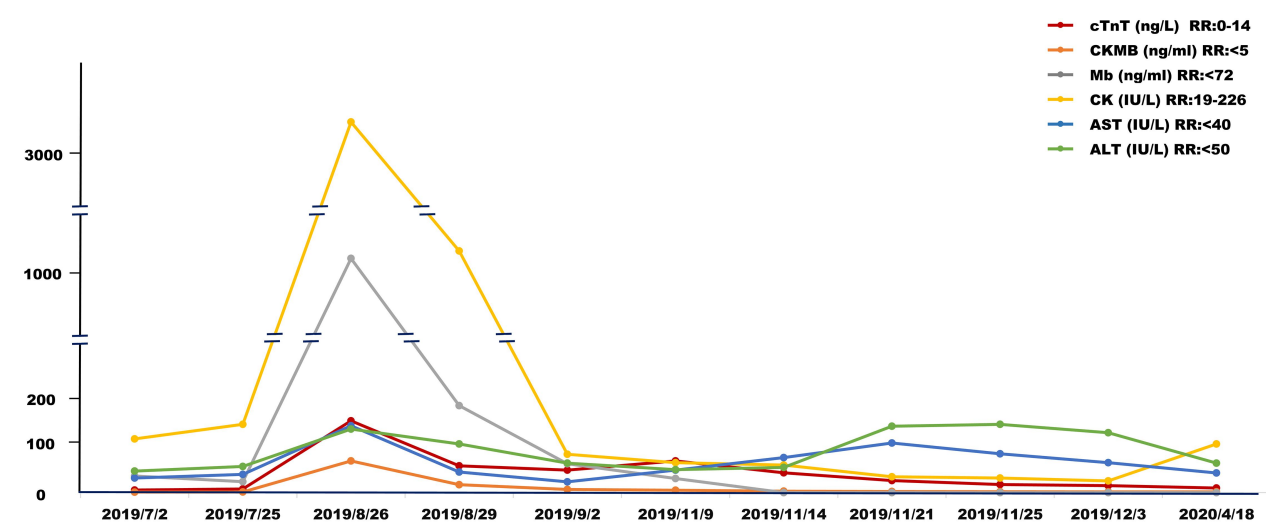

Figure 4 The dynamic change of cTnT, CKMB, Mb, CK, AST and ALT during treatment.

baseline EBV-DNA concentration in the serum is an independent poor prognostic marker in LELC patients. $^{24,25}$ And it was suggested that EBV infection might be a biomarker of favorable outcomes in immunotherapy. Kim showed that PD-L1 expression in patients with EBV-positive non-Hodgkin's lymphoma was significantly higher than EBV-negative (56\% vs $11 \%, \mathrm{p}<0.001)$, and 7 EBV-positive patients achieved survival benefits after pembrolizumab treatment. ${ }^{26}$ Kang et al proved hepatocellular carcinomas with EBV-positive tumor-infiltrating lymphocytes had a better outcome. ${ }^{27}$

\section{Tumor Microenvironment}

EBV-related LELC has a unique tumor microenvironment. Tumor antigens, either by EBV or EBV induced-cell products, recruit tumor infiltrating lymphocytes (TILs) inside the tumor stroma. ${ }^{7}$ In one report, in $84.7 \%$ of patients the staining of TILs accounted for $\geq 50 \%$. ${ }^{8}$ There had been reports TILs, either in an activated or suppressed status, contributed to a better outcome in immunotherapy with LELC. $^{28}$

\section{PD-LI Expression}

Studies showed a high level of PD-L1 expression in this tumor. Chang evaluated 66 patients of LELC and the PDL1 expression positivity (defined as $>5 \%$ ) was $75.8 \%{ }^{29}$ Similar PD-L1 expression (> 5\%) rate of $65.7 \%$ was reported by $\mathrm{Yu}(\mathrm{n}=67) .{ }^{30}$ In another study, a high level of PD-L1 expression ( $\geq 50 \%)$ was reported in $61 \%(36 / 59)$ of patients. $^{8}$ High PD-L1 expression is generally associated with good response to PD-1/PD-L1 inhibition. All these data implicated LELC might be a good candidate for ICI therapy.

Previously, 7 reports observed the efficacy of ICI in advanced LELC. Six of them were case reports (Table 2), and the final one consisted of 2 patients. ${ }^{17-22}$ Kim firstly reported a LELC patient whose PD-L1 and EBER were both positive had the tumor controlled for only 1 month after nivolumab therapy. ${ }^{18}$ All the other papers reported a good prognosis after ICI treatment.

Compared with the aforementioned anecdotal case series, our study consisted of a relatively large number of patients $(n=10)$. Also, we compared the efficacy of ICI therapy with that of traditional chemotherapy. Although the cohort of patients in ICI group was in a disadvantageous state (both up-front and salvage), the response depth was better and the PFS was much longer. Our results strongly supported ICI in these patients.

The current study had its limitation. It was based on a retrospective cohort from a single institute, and contained a limited number of patients. This was due to the scarcity of LELC, and also only recent advent of ICI in clinic. However, compared with previous case reports, our study presented a series of patients and the data might be more convincing. Secondly, ICI and Chemo group were unbalanced. Although more patients in the ICI group were in the late lines of therapy, they still had a better efficacy than those in the Chemo group. This might further support the efficacy of ICI in this group of patients.

In summary, our study provided a series of patients of LELC successfully treated with ICI. This study implicated the high efficiency of ICI therapy in this disease, especially for those with high PD-L1 expression. Prospective 


\begin{tabular}{|c|c|c|c|c|c|c|c|c|c|c|}
\hline $\begin{array}{l}\text { 䓀 } \\
\text { Qิ }\end{array}$ & 紜 & $\stackrel{\mathscr{\nu}}{\check{\nu}}$ & $\stackrel{\circ}{z}$ & 우 & $\check{\beth}^{\tilde{U}}$ & $\check{\varpi}^{\mathscr{y}}$ & ㅇ & 号 & 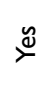 & 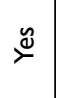 \\
\hline 乞ิ $\widehat{\xi}$ & $\stackrel{\mathscr{c}}{Z}$ & $\stackrel{m}{\infty}$ & $\widetilde{\sigma}$ & น̊ & $\stackrel{\infty}{\sim}$ & $\stackrel{\infty}{\sim}$ & $\underline{\varrho}$ & $\frac{o}{Z}$ & $\stackrel{\alpha}{z}$ & $\stackrel{\alpha}{Z}$ \\
\hline 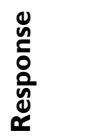 & $\stackrel{\alpha}{\alpha}$ & Q & $\stackrel{\mathscr{a}}{\alpha}$ & 只 & 只 & $\stackrel{\mathscr{I}}{\alpha}$ & $\begin{array}{l}\frac{\alpha}{\alpha} \\
\stackrel{\alpha}{\alpha}\end{array}$ & 呙 & 呙 & 只 \\
\hline ŭ $\widehat{\underline{\xi}}$ & $\stackrel{\mathscr{c}}{z}$ & - & $\stackrel{\sim}{\sim}$ & $\hat{\sim}$ & $\wedge$ & $\sigma$ & 늠 & - & $\frac{o}{z}$ & $\frac{o}{z}$ \\
\hline $\begin{array}{l}\frac{\widehat{a}}{\sqrt{5}} \\
\frac{0}{0} \\
\stackrel{5}{F}\end{array}$ & 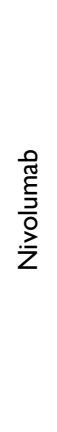 & $\begin{array}{l}\stackrel{0}{\tilde{\Xi}} \\
\underline{\underline{z}} \\
\stackrel{\partial}{z} \\
\dot{z}\end{array}$ & 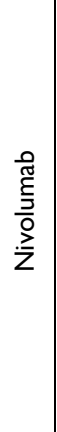 & 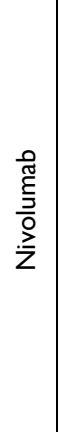 & 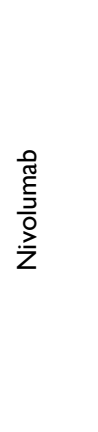 & 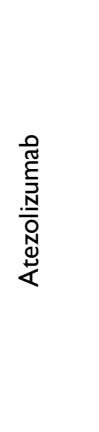 & 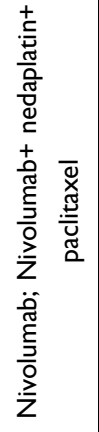 & 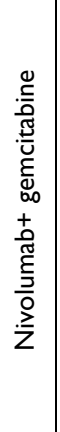 & 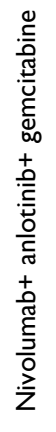 & 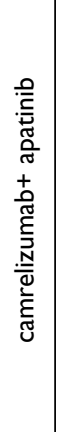 \\
\hline & 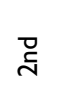 & $\stackrel{\vec{c}}{\sim}$ & $\frac{5}{f}$ & $\stackrel{0}{m}$ & $\stackrel{\text { J }}{N}$ & D. & 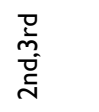 & $\underline{\ddot{n}}$ & $\underline{\ddot{n}}$ & $\stackrel{D}{D}$ \\
\hline 㽞 & + & + & + & + & + & + & + & $\frac{o}{z}$ & $\stackrel{\alpha}{z}$ & $\stackrel{o}{z}$ \\
\hline J & ○ें & + & $\frac{\mathscr{c}}{z}$ & ஸे & I & ○ें & ○ & 今ें & ○ें & 을 \\
\hline $\begin{array}{l}\stackrel{0}{ \pm} \\
\text { ஸे }\end{array}$ & 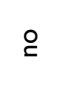 & 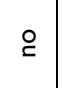 & $\stackrel{\circ}{\simeq}$ & $\stackrel{\frac{\alpha}{Z}}{Z}$ & 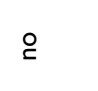 & $\stackrel{\circ}{\check{c}}$ & $\stackrel{\circ}{\check{1}}$ & $\frac{\alpha}{z}$ & $\stackrel{\alpha}{Z}$ & 受 \\
\hline $\begin{array}{l}\stackrel{\Xi}{ \pm 0} \\
\mathbb{\Xi} \\
\omega\end{array}$ & $\stackrel{\nwarrow}{\geq}$ & $\stackrel{\infty}{\geq}$ & $\stackrel{\infty}{\geq}$ & $\frac{N}{z}$ & $\stackrel{\infty}{\geq}$ & 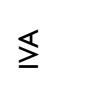 & $\stackrel{\infty}{\geq}$ & $\cong$ & $\stackrel{\varrho}{\equiv}$ & $\stackrel{\nwarrow}{\geq}$ \\
\hline 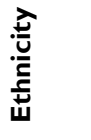 & 离 & $\frac{5}{\frac{c}{5}}$ & 蛋 & 胥 & 愛 & 离 & $\frac{\sqrt{\frac{\pi}{3}}}{\frac{0}{\alpha}}$ & 宽 & 量 & 趂 \\
\hline 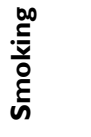 & 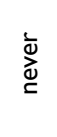 & 产 & 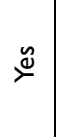 & 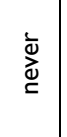 & $\stackrel{\check{\Perp}}{\check{\nu}}$ & 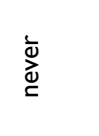 & 号 & 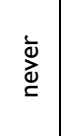 & 离 & 号 \\
\hline 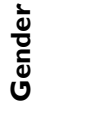 & 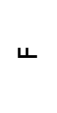 & ч & $\Sigma$ & ч & ч & ч & 4 & ч & 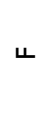 & $\Sigma$ \\
\hline$\stackrel{8}{\stackrel{\Delta}{\alpha}}$ & 亡ீ & $\hat{m}$ & 亡ீ & $\hat{m}$ & $\bar{n}$ & $\stackrel{0}{r}$ & 움 & ํำ & $\dot{q}$ & $\stackrel{\infty}{+}$ \\
\hline 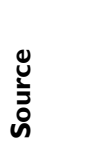 & $\begin{array}{l}\bar{\sigma} \\
\stackrel{\Delta}{0} \\
. \bar{\partial}\end{array}$ & $\begin{array}{l}\bar{\sigma} \\
\stackrel{\Delta}{0} \\
\underline{\underline{y}}\end{array}$ & 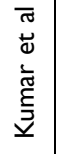 & & 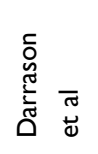 & 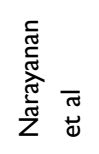 & 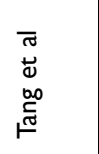 & 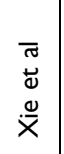 & & \\
\hline
\end{tabular}


randomized studies are warranted to confirm this conclusion.

\section{Disclosure}

The authors report no conflicts of interest in this work.

\section{References}

1. Ho JC, Wong MP, Lam WK. Lymphoepithelioma-like carcinoma of the lung. Respirology. 2006;11(5):539-545. doi:10.1111/j.1440-1843. 2006.00910.x

2. Hu Y, Ren S, Liu Y, et al. Pulmonary Lymphoepithelioma-like carcinoma: a mini-review. Onco Targets Ther. 2020;13(undefined):39213929. doi:10.2147/OTT.S241337

3. Liang Y, Wang L, Zhu Y, et al. Primary pulmonary lymphoepithelioma-like carcinoma: fifty-two patients with long-term follow-up. Cancer. 2012;118(19):4748-4758. doi:10.10 02/cncr.274.52

4. Qin Y, Gao G, Xie X, et al. Clinical features and prognosis of pulmonary lymphoepithelioma-like carcinoma: summary of eighty-five cases. Clin Lung Cancer. 2019;20(3):e329-e337. doi:10.1016/j.cllc.2018.12.014

5. Sathirareuangchai S, Hirata K. Pulmonary Lymphoepithelioma-like carcinoma. arch. Pathol Lab Med. 2019;143(8):1027-1030. doi:10.5858/arpa.2018-0149-RS

6. Bégin LR, Eskandari J, Joncas J, et al. Epstein-Barr virus related lymphoepithelioma-like carcinoma of lung. J Surg Oncol. 1987;36 (4):280-283. doi:10.1002/jso.2930360413

7. Chang YL, Wu CT, Shih JY, et al. New aspects in clinicopathologic and oncogene studies of 23 pulmonary lymphoepithelioma-like carcinomas. Am J Surg Pathol. 2002;26(6):715-723. doi:10.1097/ 00000478-200206000-00004

8. Wu Q, Wang W, Zhou P, et al. Primary pulmonary lymphoepithelioma-like carcinoma is characterized by high PD-L1 expression, but low tumor mutation burden. Pathol Res Pract. 2020;216(8):153043. doi:10.1016/j.prp.2020. 153043

9. Chan JK, Hui PK, Tsang WY, et al. Primary lymphoepithelioma-like carcinoma of the lung. A clinicopathologic study of 11 cases. Cancer. 1995;76(3):413-422. doi:10.1002/1097-0142(19950801)76:3<413: aid-cncr2820760311>3.0.co;2-x

10. Tay CK, Chua YC, Takano A, et al. Primary pulmonary lymphoepithelioma-like carcinoma in Singapore. Ann Thorac Med. 2018;13(1):30-35. doi:10.4103/atm.ATM_304_17

11. Chang YL, Wu CT, Shih JY, et al. Unique 553 and epidermal growth factor receptor gene mutation status in 46 pulmonary lymphoepithelioma-like carcinomas. Cancer Sci. 2011;102(1):282-287. doi:10.1111/j.13497006.2010.01768.x

12. Liu Q, Ma G, Yang H, et al. Lack of epidermal growth factor receptor gene mutations in exons 19 and 21 in primary lymphoepithelioma-like carcinoma of the lung. Thorac Cancer. 2014;5(1):63-67. doi:10.1111/ 1759-7714.12060

13. Xie Z, Liu L, Lin X, et al. A multicenter analysis of genomic profiles and PD-L1 expression of primary lymphoepithelioma-like carcinoma of the lung. Mod Pathol. 2020;33(4):626-638. doi:10.1038/s41379019-0391-9

14. Chan AT, Teo PM, Lam KC, et al. Multimodality treatment of primary lymphoepithelioma-like carcinoma of the lung. Cancer. 1998;83 (5):925-929. doi:10.1002/(sici)1097-0142(19980901)83:5<925::aidcncr18>3.0.co;2-x
15. Lin $\mathrm{CY}$, Chen YJ, Hsieh MH, et al. Advanced primary pulmonary lymphoepithelioma-like carcinoma: clinical manifestations, treatment, and outcome. J Thorac Dis. 2017;9(1):123-128. doi:10.21 037/jtd.2017.01.25

16. Huang CJ, Feng AC, Fang YF, et al. Multimodality treatment and long-term follow-up of the primary pulmonary lymphoepithelioma-like carcinoma. Clin Lung Cancer. 2012;13 (5):359-362. doi:10.1016/j.cllc.2012.01.002

17. Qiu ZX, Zhou P, Wang K. Primary pulmonary lymphoepithelioma-like carcinoma response favorably to nivolumab: a case report. Onco Targets Ther. 2019;12(undefined):8595-8600. doi:10.2147/OTT.S219512

18. Kim C, Rajan A, DeBrito PA, et al. Metastatic lymphoepithelioma-like carcinoma of the lung treated with nivolumab: a case report and focused review of literature. Transl Lung Cancer Res. 2016;5(6):720-726. doi:10.21037/tlcr.2016.11.06

19. Kumar V, Dave V, Harris J, et al. Response of advanced stage recurrent lymphoepithelioma-like carcinoma to nivolumab. Immunotherapy. 2017;9(12):955-961. doi:10.2217/imt-2017-0067

20. Darrason M, Martin A, Soussan M, et al. Immunotherapy for LELC: case report and a focused review. Clin Lung Cancer. 2019;20(3): e393-e401. doi:10.1016/j.cllc.2018.12.008

21. Narayanan A, Knollmann FD, Walby JAS, et al. EBV-positive primary pulmonary lymphoepithelioma-like carcinoma response to PD-L1 blockade. Clin Lung Cancer. 2019;20(3):e238-e241. doi:10.1016/j.cllc.2018.12.015

22. Tang Z, Fang R, Tong G, et al. Overcoming resistance to anti-PD-1 immunotherapy in lymphoepithelioma-like carcinoma: a case report and review of the literature. Lung Cancer. 2020;146 (undefined):335-340. doi:10.1016/j.lungcan.2020.06.027

23. Lin Z, Fu S, Zhou Y, et al. First-line platinum-based chemotherapy and survival outcomes in locally advanced or metastatic pulmonary lymphoepithelioma-like carcinoma. Lung Cancer. 2019;137 (undefined):100-107. doi:10.1016/j.lungcan.2019.09.007

24. Xie $\mathrm{M}, \mathrm{Wu} \mathrm{X}$, Wang $\mathrm{F}$, et al. Clinical significance of plasma epstein-barr virus DNA in pulmonary Lymphoepithelioma-like Carcinoma (LELC) patients. J Thorac Oncol. 2018;13(2):218-227. doi:10.1016/j.jtho.2017.10.031

25. Hong S, Liu D, Luo S, et al. The genomic landscape of Epstein-Barr virus-associated pulmonary lymphoepithelioma-like carcinoma. Nat Commun. 2019;10(1):3108. doi:10.1038/s41467-019-10902-w

26. Kim SJ, Hyeon J, Cho I, et al. Comparison of efficacy of pembrolizumab between Epstein-Barr virus-positive and -negative relapsed or refractory non-hodgkin Lymphomas. Cancer Res Treat. 2019;51 (2):611-622. doi:10.4143/crt.2018.191

27. Kang HJ, Oh JH, Chun SM, et al. Immunogenomic landscape of hepatocellular carcinoma with immune cell stroma and EBV-positive tumor-infiltrating lymphocytes. $J$ Hepatol. 2019;71(1):91-103. doi:10.1016/j.jhep.2019.03.018

28. Kasai K, Kon S, Sato N, et al. Case report of lymphoepithelioma-like carcinoma of the lung-lymphoid population consisting of cytotoxic T cells in resting state. Pathol Res Pract. 1999;195(11):773-779. doi:10.1016/S0344-0338(99)80120-4

29. Chang YL, Yang CY, Lin MW, et al. PD-L1 is highly expressed in lung lymphoepithelioma-like carcinoma: a potential rationale for immunotherapy. Lung Cancer. 2015;88(3):254-259. doi:10.1016/j. lungcan.2015.03.017

30. Yu XY, Zhang XW, Wang F, et al. Correlation and prognostic significance of PD-L1 and P53 expression in resected primary pulmonary lymphoepithelioma-like carcinoma.J Thorac Dis. 2019;10 (3):1891-1902. DOI:10.21037/jtd.2018.03.14 


\section{Publish your work in this journal}

OncoTargets and Therapy is an international, peer-reviewed, open access journal focusing on the pathological basis of all cancers, potential targets for therapy and treatment protocols employed to improve the management of cancer patients. The journal also focuses on the impact of management programs and new therapeutic

Submit your manuscript here: https://www.dovepress.com/oncotargets-and-therapy-journa/ agents and protocols on patient perspectives such as quality of life, adherence and satisfaction. The manuscript management system is completely online and includes a very quick and fair peer-review system, which is all easy to use. Visit http://www.dovepress.com/ testimonials.php to read real quotes from published authors. 